\title{
Editorial for the special section
}

\section{Cognitive neuroscience: behavioral and neuroimaging studies on language processing}

\author{
Rochele Paz Fonseca ${ }^{1}$ Lilian Cristine Scherer ${ }^{2,3}$ \\ 1 Pontifícia Universidade Católica do Rio Grande do Sul, Brazil \\ 2 Université de Montréal, Canada \\ 3 Universidade de Santa Cruz do Sul, Brazil
}

Cognitive neuroscience comprises a wide field of investigation, encompassing an array of complementary domains. Among these domains is neuropsychology, which emerged from lesion studies, and its relationship to language, first reported in 1861 by the French neurologist Paul Broca. This field has been increasingly developed worldwide. In this context, this special issue of Psychology \& Neuroscience, entitled "Language and Cognitive Neuroscience," aims to present updated manuscripts on theoretical, behavioral, and neuroimaging data derived from language studies.

A very important field of research in neuropsychology is neuropsychological assessment. This research domain has a strong interface with neuropsycholinguistics, because of the fact that language is both the vehicle to convey the assessment and the focus of investigation in verbal instruments, meaning that language is used to evaluate language itself. Despite its importance to cognitive neuroscience and to this subarea of neuropsychological assessment, language appears not to be one of the most studied cognitive processes by neuropsychologists. In the 1970s, relevant psycholinguistic models emerged, including pragmatics and discourse processing theories, which proposed an analysis of language beyond its basic structural facets. For example, the speech acts model, proposed by Searle (1969), and the text organization model, proposed by van Dijk and Kintsch (1978), served as the basis for theories on pragmatics and discourse adopted today. Although these models could be an important theoretical basis for the assessment of language production and comprehension, language remains a topic scarcely studied by neuropsychologists compared with other cognitive processes.

Among the sources of knowledge about language processing are experiments, quasi-experiments, and case studies that use healthy samples and neurological or psychiatric subjects that provide behavioral data from standardized general cognitive or language evaluation tools and neuroimaging data from structural and functional neuroimaging techniques. Importantly, the investigation of impaired language processing may shed light on our understanding of brain plasticity by studying the neurobiological and functional reorganization of language circuitry following a lesion. Another research field encompassed by language studies is bilingualism, a complex linguistic phenomenon that deserves thorough investigation because of the bilingual characteristics of the majority of the world's population. Finally, by studying language production and comprehension, researchers may garner important insights into the functioning of several cognitive abilities intrinsically related to language processing, such as working memory, attention, and several executive functions.

The emergence of structural and functional neuroimagingtechniques, suchascomputerizedtomography (CT), magnetic resonance imaging (MRI), positron emission tomography(PET) and magnetoencephalography (MEG), has allowed researchers to gather more precise and sophisticated data on how cognitive processing occurs in the brain. Studies on cognition, including language, underwent incredible growth, especially since the 1990s, a decade known as the "decade of the brain.” Neuroimaging techniques rendered the study of neurofunctional human brain circuitry possible in vivo, which transformed studies from being merely speculative to more data-driven.

Moreover, the results obtained from these studies allowed neuropsychologists and other researchers from related fields to compare the formerly established theories on cognitive processing and their behavioral results of language performance with the new neuroimaging evidence, thus either comoborating these theories or allowing the proposal of updated or new ones. Thus, an enormous advance in our knowledge about human cognition has been afforded by the ever-refined neuroimaging techniques available to researchers. 
This special edition of Psychology \& Neuroscience on language and cognitive neuroscience illustrates some of these studies. This section covers normal and impaired language processing from studies ranging from the word to discourse level in monolingual and bilingual populations. The article by Ska and collaborators provides theoretical, behavioral, and neuroimaging data on discourse processing, discussing these aspects within the framework of a review that covers recent studies from the scarcely studied field of discourse processing. An fMRI study developed by Buchweitz and colleagues discusses the effects of presentation modality-oral and written-and the influence of individual differences in language comprehension. Another article, by Waldie and colleagues, focuses on a research field in language processing, namely bilingualism, that offers a window into the understanding of language organization and functioning in the brain. This article reports an fMRI study investigating the performance of late-proficient bilinguals in a demanding executive function and attention control task. An ERP study investigating the process of young adults drawing inferences while reading two types of text, narrative and expository, is presented by Baretta and colleagues. A fifth article, by Brandão and collaborators, presents results from a study on cognition and discourse production in Alzheimer's disease by the use of informative prompts. Finally, an instrument to quickly and reliably classify aphasic patient symptoms in syndromes based on cognitive assessment is the focus of the article by Wilson and colleagues.

With the constant development of evidence-based cognitive neuroscience, our knowledge about different components and levels of language processing has grown appreciably. Behavioral data have been extensively explored, but their relationship to neuroimaging data still needs to be the focus of future investigations. This special section of Psychology \& Neuroscience seeks to move toward elucidating these incipient relationships.

\section{References}

Kintsch, W., \& van Dijk, T.A. (1978). Towards a model of text comprehension and production. Psychological Review, 85, 363-394.

Searle, J.R. (1969). Speech acts: an essay in the philosophy of language. Cambridge: Cambridge University Press. 